\section{A coordenação do cuidado na qualidade da assistência à saúde da mulher e da criança no PMAQ}

\section{Coordination of care and quality of healthcare for women and children in the PMAQ}

\section{La coordinación del cuidado en la calidad de la asistencia a la salud de la mujer y del niño en el PMAQ}

\section{Resumo}

O objetivo foi identificar a associação do atributo coordenação do cuidado com a qualidade da assistência à saúde da mulher e da criança na atenção primária à saúde no Brasil. Foi realizado estudo transversal, baseado em dados de 30.523 equipes que participaram do Programa de Melhoria do Acesso e da Qualidade da Atenção Básica em 2013. Foi feita análise de regressão logística. A variável dependente foi o nível de qualidade da assistência à saúde da mulher e da criança, e a independente, o nível de coordenação do cuidado. $A$ análise multivariada considerou variáveis que apresentaram $p<0,05$. $O$ ajuste do modelo foi realizado pelo teste de Hosmer-Lemeshow. Foram avaliados os resultados de 28.056 equipes que realizaram atividade de coordenação e de assistência à saúde da mulher e da criança simultaneamente. No Brasil, o maior percentual das equipes apresentou nivel baixo de coordenação $(68,5 \%)$. Os níveis mais altos de coordenação foram encontrados no estrato $6(57,2 \%) e$ os mais baixos no estrato 1 (78,5\%). Dentre as regiões, a Norte apresentou o maior percentual de equipes com baixo nível de coordenação (89,1\%), e a Sudeste, o maior percentual com alto nível (37,6\%). Para o nível de qualidade da assistência à saúde da mulher, 70,5\% das equipes estava com baixo nível e, na saúde da criança, 63,5\% com alto nível. Possuir alto nível de coordenação está associado a ter alto nível de qualidade da assistência, tanto na saúde da mulher $(O R=11,85)$ como na saúde da criança $(O R=8,79)$. Foi possível constatar um predomínio de baixos níveis de coordenação do cuidado no Brasil, bem como baixos níveis de qualidade da assistência à saúde da mulher, refletindo a necessidade de ações coordenadas nessa área.

Atenção Primária à Saúde; Saúde da Criança; Saúde da Mulher; Garantia da Qualidade dos Cuidados de Saúde; Pesquisa sobre Serviços de Saúde
Maria Jesus Barreto Cruz 1

Alaneir de Fátima dos Santos 1

Lucas Henrique Lobato de Araújo 1

Eli Iola Gurgel Andrade 1

doi: 10.1590/0102-311X00004019

\section{Correspondência}

M. J. B. Cruz

Universidade Federal de Minas Gerais.

Av. Prof. Alfredo Balena 190, Belo Horizonte, MG 30130-100, Brasil.

maria_enfermagem@yahoo.com.br

1 Universidade Federal de Minas Gerais, Belo Horizonte, Brasil. 


\section{Introdução}

Serviços de atenção primária à saúde (APS) estruturados e coordenadores do cuidado são mais eficazes no gerenciamento da atenção e na oferta adequada de ações em consonância com as reais necessidades da população. Em todas as áreas da assistência à saúde, a coordenação do cuidado é fundamental, estando relacionada a melhores resultados, seja na adequada alocação dos recursos públicos, seja na satisfação dos usuários 1. Esse atributo tem sido apontado como um importante mecanismo para reduzir as barreiras de acesso, oferecendo uma assistência mais integrada e eficiente, que beneficia a estruturação do sistema de modo geral 2.

Para a saúde da mulher e da criança, o componente de coordenação do cuidado tem grande representatividade na busca por maior qualidade da atenção e, por isso mesmo, constitui-se um grande desafio. Esse tema tem ampla repercussão mundial, e esforços têm sido empregados para melhorar as condições de saúde e reduzir a morbimortalidade nessas populações 3 . Contudo, indícios de descontinuidade na assistência ainda podem ser identificados com certa frequência, refletidos em níveis ainda altos de mortalidade materna e infantil 4.

No que se refere ao atributo coordenação do cuidado, a Organização Mundial da Saúde (OMS) reconhece que, quando viabilizado por uma rede de serviços, é capaz de ofertar uma resposta integral e articulada em todos os níveis de atenção 5 . Ao ser entendido como a articulação entre os serviços de saúde, independentemente do local onde sejam prestados, pode garantir a sincronização em torno de um mesmo objetivo ${ }^{6}$. Envolve, ademais, a realização de ações que conjugam o planejamento da assistência e de fluxos, o compartilhamento de informações sobre o processo assistencial entre as redes de atenção e o monitoramento de pacientes com diferentes necessidades 7 .

Em modelo proposto por McDonald et al. ${ }^{8}$, a coordenação do cuidado está no centro do processo e implica a organização deliberada de atividades que envolvem usuário do serviço, profissionais de saúde e o próprio sistema de saúde. A coordenação do cuidado, portanto, desempenha a função de elo entre todos os mecanismos envolvidos no cuidado, como recursos da comunidade, atendimentos assistenciais, dentre outros 9 .

Quanto mais ampla a rede de pessoas e serviços envolvidos no processo de cuidado, e quanto maior a complexidade das intervenções a serem realizadas, maior será o nível de coordenação para a resolução almejada 8 . As ações de coordenação, dessa forma, devem ser entendidas em uma perspectiva dinâmica, como algo que se adapta ao perfil do sistema, bem como às especificidades dos grupos e indivíduos que demandam o cuidado e a oferta de serviços 9 . O resultado de ações bem coordenadas contribui para a redução de erros de diagnóstico e de tratamento, de filas de espera, de duplicações de provas diagnósticas e de hospitalizações desnecessárias 2,10.

Em âmbito nacional ou internacional, o alcance desses atributos é entendido hoje como parâmetro para avaliar a robustez do sistema de atenção 11. Essa conscientização tem conduzido à necessidade de avaliação, seja de maneira isolada, seja dentro do sistema como um todo. Por ser um produto de várias organizações, pelas questões teóricas que envolvem a definição do conceito ou pela dificuldade em distingui-la de outros aspectos ou de processos, avaliar a coordenação do cuidado é uma tarefa complexa 12. Tais dificuldades têm limitado o entendimento da influência da coordenação nos resultados de avaliações de políticas e ações de saúde, assim como têm impedido avanços diretos no cuidado 13 .

Na busca constante para promover a evolução desse atributo, a Agência de Pesquisa e Qualidade em Saúde (Agency for Healthcare Research and Quality - AHRQ) dos Estados Unidos desenvolveu em 2011, com revisão em 2014, o Atlas de Medidas de Coordenação de Cuidados 13. Trata-se de um instrumento que permite identificar medidas adequadas para avaliar as intervenções de coordenação de cuidados, considerando as perspectivas de pacientes, cuidadores, profissionais de saúde e sistemas de saúde 8 . É na atualidade um instrumento indispensável para pesquisadores da saúde, assim como para gestores e instituições que buscam a melhoria no acesso e na qualidade da atenção.

O Observatório Europeu dos Sistemas e Políticas de Saúde também vem desenvolvendo relevantes pesquisas nessa direção. Em 2015, foi divulgado resultado de trabalho realizado pelo Monitor de Atividade de Atenção Primária à Saúde para a Europa (Primary Health Care Activity Monitor for Europe - PHAMEU), que forneceu o mapeamento e a comparação dos sistemas de APS para 31 países no continente, com descrição para variações de acessibilidade, continuidade e coordenação, com dimensões e inter-relações entre eles. A coordenação do cuidado foi identificada na dimensão relativa 
ao processo, com foco na prestação de serviços, refletindo a capacidade de coordenação da APS em todos os níveis assistenciais para cada país 2 . Esse trabalho fornece elementos importantes para a avaliação da coordenação do cuidado na APS, podendo ser subsídio para desenvolvimento de pesquisas em muitos outros países, sobretudo naqueles que adotam esse modelo de atenção.

No Brasil, diversos esforços vêm sendo realizados rumo ao acesso a serviços de saúde de qualidade. Políticas públicas, como Requalifica UBS (unidades básicas de saúde), Programa Mais Médicos (PMM), Programa Nacional de Melhoria do Acesso e da Qualidade da Atenção Básica (PMAQ), bem como ações para ampliação da saúde bucal e dos Núcleos de Apoio à Saúde da Família (NASF) contribuem para o fortalecimento de processos assistenciais na APS 14. Nessa perspectiva, o PMAQ desempenha um importante papel ao introduzir de forma ampla a avaliação de desempenho da APS 15. Esse programa tem como objetivo induzir a ampliação do acesso e a melhoria da qualidade da atenção, além de estimular o aprimoramento de padrões e indicadores de qualidade da saúde 16.

No entanto, estudo realizado por Fausto et al. 17 evidencia que as estratégias para integração da rede assistencial e coordenação do cuidado no sistema brasileiro ainda são incipientes, como também ainda são escassas as pesquisas na área de avaliação organizacional ou de desempenho da APS, dotadas de visão crítica dos mecanismos institucionais de monitoramento e avaliação 18.

Diante do exposto, o objetivo deste artigo é identificar a associação do atributo coordenação do cuidado com a qualidade da assistência à saúde da mulher e da criança na APS no Brasil.

\section{Métodos}

Estudo transversal, com abordagem quantitativa, com base no banco de dados do segundo ciclo do PMAQ, realizado no ano 2013, em 30.523 equipes de atenção básica distribuídas em todo o território nacional.

Neste estudo, as atividades de coordenação do cuidado e referentes à saúde da mulher e da criança realizadas pelas equipes avaliadas foram identificadas no conjunto de variáveis do instrumento de avaliação externa do Módulo II, que contém a entrevista do profissional sobre o processo de trabalho da equipe e a organização do cuidado, além da verificação de documentos relacionados à unidade de saúde.

Para a análise da coordenação do cuidado, foram consideradas as seguintes dimensões: Gestão Municipal para o Desenvolvimento da Atenção Primária, Valorização do Trabalhador e Acesso e Qualidade da Atenção e Organização do Processo de Trabalho. Para a análise da qualidade da atenção à saúde da mulher e da criança, foi considerada somente a Dimensão Acesso e Qualidade da Atenção e Organização do Processo de Trabalho. As subdimensões e o número de variáveis utilizadas estão descritos no Quadro 1.

Para a análise, foram consideradas as 30.523 equipes que participaram do 2o ciclo do PMAQ. Inicialmente foram identificadas as equipes que realizaram atividades de coordenação do cuidado e, dentre essas, as que relataram ter realizado alguma atividade de coordenação do cuidado e de assistência à saúde da mulher e da criança, totalizando 28.056 equipes. Portanto, o estudo contemplou as equipes que possuíam simultaneamente as duas características. Foram criadas tipologias de coordenação do cuidado abrangendo as atividades de coordenação realizadas pelas equipes, bem como tipologias de qualidade da assistência à saúde da mulher e da criança abrangendo as atividades de assistência oferecidas a esses grupos.

O nível de coordenação foi estimado pela razão entre o somatório das atividades de coordenação do cuidado desenvolvidas pelas equipes e o total de questões referentes à coordenação, sem ponderação de pesos entre as variáveis. As questões referentes à coordenação do cuidado foram identificadas no instrumento de avaliação externa e considerou-se o conceito amplo de coordenação do cuidado, tendo como referência estudos nacionais e internacionais que avaliaram o atributo 2,8,19,20. Assim, as atividades consideradas como coordenação do cuidado que direcionaram a escolha das questões foram: ações e mecanismos de comunicação; apoio comunitário; apoio para tomada de decisão; compartilhamento de informações entre os envolvidos no processo de cuidado; estabelecimento e/ou compartilhamento de responsabilidade no cuidado; estratificação de risco do território e das condições de atenção; implantação e utilização do e-SUS e prontuário eletrônico; mecanismos de 


\section{Quadro 1}

Subdimensões utilizadas para a análise da coordenação do cuidado e saúde da mulher e da criança.

\begin{tabular}{|c|c|c|c|}
\hline & Subdimensão & $\begin{array}{l}\text { Total de questões } \\
\text { (selecionadas) }\end{array}$ & Atividades identificadas \\
\hline \multirow{17}{*}{$\begin{array}{l}\text { Coordenação } \\
\text { de cuidado }\end{array}$} & $\begin{array}{l}\text { Educação permanente do } \\
\text { processo de qualificação das } \\
\text { ações desenvolvidas }\end{array}$ & $12(2)$ & $\begin{array}{l}\text { Ações e mecanismos de comunicação; compartilhamento de } \\
\text { informações; educação permanente; estabelecimento e/ou } \\
\text { compartilhamento de responsabilidade no cuidado. }\end{array}$ \\
\hline & $\begin{array}{l}\text { Planejamento da equipe de } \\
\text { atenção básica e ações da gestão } \\
\text { para a organização do processo } \\
\text { de trabalho da equipe }\end{array}$ & $18(9)$ & $\begin{array}{l}\text { Ações e mecanismos de comunicação; apoio para tomada de decisão; } \\
\text { compartilhamento de informações entre os envolvidos no processo } \\
\text { de cuidado; estratificação de risco do território e das condições de } \\
\text { atenção; uso de sistemas de informação; reuniões; utilização de } \\
\text { protocolos clínicos. }\end{array}$ \\
\hline & $\begin{array}{l}\text { Organização dos prontuários na } \\
\text { unidade de saúde }\end{array}$ & $11(11)$ & $\begin{array}{l}\text { Implantação e utilização do e-SUS e prontuário eletrônico, uso de } \\
\text { sistemas de informação. }\end{array}$ \\
\hline & $\begin{array}{l}\text { Acolhimento à demanda } \\
\text { espontânea }\end{array}$ & $21(4)$ & $\begin{array}{c}\text { Apoio para tomada de decisão; utilização de protocolos clínicos; } \\
\text { organização da agenda. }\end{array}$ \\
\hline & Organização da agenda & $6(5)$ & $\begin{array}{l}\text { Mecanismos de referência e contrarreferência; organização da } \\
\text { agenda. }\end{array}$ \\
\hline & Atenção à saúde & $8(8)$ & $\begin{array}{l}\text { Apoio comunitário; estratificação de risco do território e das } \\
\text { condições de atenção; mecanismos de referência e contrarreferência; } \\
\text { registro de informações, implantação e utilização do e-SUS e } \\
\text { prontuário eletrônico; utilização de protocolos clínicos. }\end{array}$ \\
\hline & $\begin{array}{l}\text { Quais exames são solicitados pela } \\
\text { equipe de atenção básica e são } \\
\text { realizados pela rede de serviços } \\
\text { de saúde }\end{array}$ & $2(1)$ & $\begin{array}{c}\text { Apoio para tomada de decisão; mecanismos de referência e } \\
\text { contrarreferência. }\end{array}$ \\
\hline & $\begin{array}{l}\text { Integração da rede de atenção à } \\
\text { saúde: ordenamento e definição } \\
\text { de fluxos }\end{array}$ & $3(3)$ & $\begin{array}{l}\text { Apoio para tomada de decisão; mecanismos de referência e } \\
\text { contrarreferência; registro de informações. }\end{array}$ \\
\hline & $\begin{array}{l}\text { Oferta e resolubilidade de ações } \\
\text { da equipe }\end{array}$ & $2(2)$ & Apoio para tomada de decisão. \\
\hline & Atenção à pessoa com obesidade & $3(3)$ & $\begin{array}{l}\text { Apoio para tomada de decisão; estratificação de risco do } \\
\text { território e das condições de atenção; mecanismos de referência } \\
\text { e contrarreferência; registro de informações, uso de sistemas de } \\
\text { informação. }\end{array}$ \\
\hline & $\begin{array}{c}\text { Atenção ao usuário em sofrimento } \\
\text { psíquico }\end{array}$ & $10(9)$ & $\begin{array}{l}\text { Apoio para tomada de decisão; estratificação de risco do território e } \\
\text { das condições de atenção; registro de informações. }\end{array}$ \\
\hline & Atenção à pessoa com deficiência & $3(3)$ & Registro de informações. \\
\hline & Programa Bolsa Família & $2(2)$ & $\begin{array}{l}\text { Estratificação de risco do território e das condições de atenção; } \\
\text { registro de informações. }\end{array}$ \\
\hline & $\begin{array}{l}\text { Visita domiciliar e cuidado } \\
\text { realizado no domicílio }\end{array}$ & $8(7)$ & $\begin{array}{l}\text { Estratificação de risco do território e das condições de atenção; } \\
\text { utilização de protocolos clínicos; registro de informações. }\end{array}$ \\
\hline & $\begin{array}{c}\text { Participação, controle social, } \\
\text { satisfação e canal de comunicação } \\
\text { com o usuário }\end{array}$ & $6(2)$ & Apoio comunitário; mecanismos de referência e contrarreferência. \\
\hline & Atividades nas escolas & $6(6)$ & $\begin{array}{l}\text { Estratificação de risco do território e das condições de atenção; } \\
\text { registro de informações; utilização de protocolos clínicos. }\end{array}$ \\
\hline & NASF & $23(16)$ & $\begin{array}{l}\text { Ações e mecanismos de comunicação; apoio para tomada de } \\
\text { decisão; compartilhamento de informações entre os envolvidos no } \\
\text { processo de cuidado; estabelecimento e/ou compartilhamento de } \\
\text { responsabilidade no cuidado; planejamento de ações. }\end{array}$ \\
\hline
\end{tabular}

(continua) 
Quadro 1 (continuação)

\begin{tabular}{|c|c|c|c|}
\hline & Subdimensão & $\begin{array}{l}\text { Total de questões } \\
\text { (selecionadas) }\end{array}$ & Atividades identificadas \\
\hline \multirow{8}{*}{$\begin{array}{l}\text { Saúde da } \\
\text { mulher }\end{array}$} & Organização da agenda & $6(2)$ & Oferta de serviços. \\
\hline & Atenção à súde & $8(10)$ & $\begin{array}{l}\text { Busca ativa; oferta de consultas; organização da agenda; protocolos } \\
\text { estratificação de risco; registros. }\end{array}$ \\
\hline & $\begin{array}{l}\text { Quais exames são solicitados pela } \\
\text { equipe de atenção básica e são } \\
\text { realizados pela rede de serviços } \\
\text { de saúde }\end{array}$ & $2(2)$ & Oferta de serviços. \\
\hline & $\begin{array}{l}\text { Oferta e resolubilidade de ações } \\
\text { da equipe }\end{array}$ & $2(2)$ & Oferta de serviços. \\
\hline & $\begin{array}{l}\text { Atenção ao pré-natal, parto e } \\
\text { puerpério }\end{array}$ & $9(13)$ & $\begin{array}{l}\text { Administração de penicilina G benzatina; monitoramento do } \\
\text { parto; oferta de consultas; registros; vacinação, uso de sistemas de } \\
\text { informação. }\end{array}$ \\
\hline & Atenção à pessoa com obesidade & $3(1)$ & Controle peso, uso de sistemas de informação. \\
\hline & Promoção da saúde & $2(2)$ & Ações educativas, planejamento familiar. \\
\hline & NASF & $23(1)$ & Apoio do NASF na área materno-infantil. \\
\hline \multirow{5}{*}{$\begin{array}{l}\text { Saúde da } \\
\text { criança }\end{array}$} & $\begin{array}{l}\text { Acolhimento à demanda } \\
\text { espontânea }\end{array}$ & $21(1)$ & Protocolos de atendimento a criança. \\
\hline & Organização da agenda & $8(1)$ & Oferta de ações. \\
\hline & Atenção à saúde & $8(4)$ & $\begin{array}{l}\text { Busca ativa; oferta de consultas; organização da agenda; protocolos } \\
\text { de estratificação de risco; registros. }\end{array}$ \\
\hline & $\begin{array}{l}\text { Atenção à criança desde o } \\
\text { nascimento até os dois anos de } \\
\text { vida }\end{array}$ & $8(16)$ & $\begin{array}{l}\text { Acompanhamento do crescimento e desenvolvimento; consultas; } \\
\text { oferta de orientações; utilização da caderneta da criança. }\end{array}$ \\
\hline & Atenção à pessoa com obesidade & $8(2)$ & Acompanhamento do peso e altura, uso de sistemas de informação. \\
\hline
\end{tabular}

NASF: Núcleo de Apoio à Saúde da Família.

referência e contrarreferência; organização da agenda; planejamento de ações; processo de trabalho entre as equipes de APS e das equipes de apoio matricial e institucional; registro de informações; reuniões, utilização de protocolos clínicos e uso de sistemas de informação. Dessa forma, para a seleção das questões, foi realizada uma busca criteriosa por questões de coordenação do cuidado, englobando desde aspectos gerenciais a oferta de cuidados em todas as áreas contempladas pelo PMAQ: saúde mental, tuberculose, hipertensão, diabetes, obesidade, hanseníase e saúde na escola.

Foram considerados os seguintes níveis: coordenação baixa (0 a 69,9\%) e coordenação alta (70 a 100\%). Adicionalmente, o nível de coordenação foi analisado segundo estratos dos municípios, e as regiões, por meio das frequências absoluta e relativa. Os estratos foram criados pelo MS, com a intenção de garantir uma maior equidade na comparação entre as equipes no processo de certificação. A definição dos estratos foi feita com base em índice que varia de 0 a 10 e porte populacional. O índice é composto de cinco indicadores: Produto Interno Bruto (PIB) per capita; percentual da população com plano de saúde; percentual da população com Bolsa Família; percentual da população em extrema pobreza e densidade demográfica 16.

Assim, os municípios são classificados e distribuídos em seis estratos, a saber: 1 = pontuação menor que 4,82 e população de até 10 mil habitantes; 2 = pontuação menor que 4,82 e população de até 20 mil habitantes; 3 = pontuação menor que 4,82 e população de até 50 mil habitantes; 4 = pontuação entre 4,82 e 5,4 e população de até 100 mil habitantes; e pontuação menor que 4,82 e população entre 50 e 100 mil habitantes; 5 = pontuação entre 5,4 e 5,85 e população de até 500 mil habitantes; e pontuação menor que 5,4 e população entre 100 e 500 mil habitantes; 6 = população acima de 500 mil habitantes ou com pontuação igual ou superior a 5,85 16 . 
O nível de qualidade da assistência à saúde da mulher e da criança foi estimado pela razão entre o somatório das atividades de assistência desenvolvidas pelas equipes e o total de questões referentes à qualidade da assistência à saúde da mulher e da criança. Foram considerados os seguintes níveis: qualidade da assistência baixa (0 a 69,9\%) e qualidade da assistência alta (70 a 100\%). Adicionalmente, o nível de qualidade da assistência à saúde da mulher e da criança foi analisado em âmbito nacional por meio das frequências absoluta e relativa. Para a análise do nível de qualidade da assistência à saúde da mulher e da criança, não foram considerados os estratos dos municípios.

Para verificar associações entre o nível de coordenação do cuidado realizado pelas equipes e a qualidade da assistência à saúde da mulher e da criança, foi feita a análise de regressão logística binária múltipla, tendo como variável dependente o nível de qualidade da assistência à saúde da mulher e da criança. A variável independente foi o nível de coordenação do cuidado. Utilizaram-se como variáveis de ajuste as regiões do Brasil (Norte, Nordeste, Sudeste, Sul e Centro-oeste) e os estratos utilizados pelo PMAQ.

Para a análise, foram adotados como nível de significância estatística $\mathrm{p}<0,05$ e intervalo de $95 \%$ de confiança (IC95\%), com cálculo da respectiva odds ratio (OR) para indicar a magnitude das associações. As estimativas de OR e IC95\% de cada variável foram ajustadas para o efeito das demais variáveis estudadas no modelo. O ajuste do modelo múltiplo final foi feito por meio do teste de Hosmer-Lemeshow. Utilizou-se o Statistical Package for the Social Sciences - SPSS versão 15.0 (https://www.ibm. $\mathrm{com} /$ ) - para as análises estatísticas.

O estudo foi aprovado pelo Comitê de Ética em Pesquisa da Universidade Federal de Minas Gerais, registro 28.804, em 30 de maio de 2012.

\section{Resultados}

O estudo incluiu 30.523 equipes que participaram do 2 o ciclo do PMAQ, tendo sido avaliados os resultados de 28.056 equipes de saúde da família que relataram ter realizado atividade de coordenação do cuidado e de assistência à saúde da mulher e da criança. No Brasil, é possível verificar que o maior percentual das equipes apresenta nível baixo de coordenação $(68,5 \%)$, seguido pelas de alto nível (31,5\%). Nos estratos, observam-se achados semelhantes, e o maior percentual de equipes apresentou nível de coordenação baixo. Chama a atenção que, no estrato 6, as equipes apresentam uma situação mais avançada de coordenação $(57,2 \%)$ em comparação aos demais que possuem nível baixo. É importante destacar que os níveis mais baixos de coordenação estão no estrato $1(78,5 \%)$ e no $2(74,7 \%)$ e, à medida em que se eleva o estrato, o percentual de equipes que apresentou nível de coordenação baixo diminui (Tabela 1).

A Tabela 2 detalha a distribuição do nível de coordenação pelas regiões brasileiras. É possível verificar que o nível de coordenação baixo foi predominante em todas as regiões. A Região Norte destaca-se por apresentar o maior percentual de equipes com baixo nível de coordenação (89,1\%), seguida pela Região Centro-oeste (84\%) e, mesmo em termos discretos, a Região Sudeste é a que apresenta o maior percentual de equipes com alto nível de coordenação (37,6\%), seguida pela Região Nordeste (33,2\%) (Tabela 2).

Para o nível de qualidade da assistência à saúde da mulher e da criança, foi feita análise dicotômica das equipes avaliadas. Foi possível perceber que as dimensões estudadas apresentaram diferentes resultados. Para a saúde da mulher, o percentual das equipes com baixo nível (70,5\%) de qualidade da assistência foi superior ao das que tiveram alto nível (29,5\%). Já a qualidade da assistência à saúde da criança se destaca com maior percentual de equipes com alto nível $(63,5 \%)$, em relação àquelas com baixo nível (36,5\%) (Tabela 3).

A Tabela 4 detalha a associação entre as dimensões avaliadas e o nível de coordenação do cuidado realizado. Verifica-se associação positiva entre o nível de coordenação alto e o nível de qualidade da assistência em ambas as dimensões. Possuir alto nível de coordenação, tanto na saúde da mulher (OR $=11,85)$ como na saúde da criança $(\mathrm{OR}=8,79)$, está associado a ter um alto nível de qualidade da assistência nessas áreas (Tabela 4). 
Tabela 1

Distribuição de equipes e níveis de coordenação do cuidado, por estrato (Programa Nacional de Melhoria do Acesso e da Qualidade da Atenção Básica - PMAQ). Brasil, 2013.

\begin{tabular}{|c|c|c|c|c|c|c|}
\hline \multirow[t]{3}{*}{ Estrato } & \multicolumn{6}{|c|}{ Nível de coordenação } \\
\hline & \multicolumn{2}{|c|}{ Baixo } & \multicolumn{2}{|c|}{ Alto } & \multicolumn{2}{|c|}{ Total } \\
\hline & $\mathrm{n}$ & $\%$ & $\mathrm{n}$ & $\%$ & $n$ & $\%$ \\
\hline 1 & 2.659 & 78,5 & 729 & 21,5 & 3.388 & 100,0 \\
\hline 2 & 3.010 & 74,7 & 1.022 & 25,3 & 4.032 & 100,0 \\
\hline 3 & 3.179 & 69,9 & 1.369 & 30,1 & 4.548 & 100,0 \\
\hline 4 & 3.594 & 68,9 & 1.625 & 31,1 & 5.219 & 100,0 \\
\hline 5 & 3.340 & 68,4 & 1.545 & 31,6 & 4.885 & 100,0 \\
\hline 6 & 3.424 & 57,2 & 2.560 & 42,8 & 5.984 & 100,0 \\
\hline Total & 19.206 & 68,5 & 8.850 & 31,5 & 28.056 & 100,0 \\
\hline
\end{tabular}

Fonte: elaboração própria.

\section{Tabela 2}

Distribuição de equipes e níveis de coordenação do cuidado, por região (Programa Nacional de Melhoria do Acesso e da Qualidade da Atenção Básica - PMAQ). Brasil, 2013.

\begin{tabular}{|c|c|c|c|c|c|c|}
\hline \multirow[t]{3}{*}{ Região } & \multicolumn{6}{|c|}{ Nível de coordenação } \\
\hline & \multicolumn{2}{|c|}{ Baixo } & \multicolumn{2}{|c|}{ Alto } & \multicolumn{2}{|c|}{ Total } \\
\hline & $n$ & $\%$ & $n$ & $\%$ & $n$ & $\%$ \\
\hline Norte & 1.797 & 89,1 & 220 & 10,9 & 2.017 & 100,0 \\
\hline Nordeste & 6.777 & 66,8 & 3.370 & 33,2 & 10.147 & 100,0 \\
\hline Sudeste & 5.917 & 62,4 & 3.570 & 37,6 & 9.487 & 100,0 \\
\hline Sul & 2.938 & 68,5 & 1.351 & 31,5 & 4.289 & 100,0 \\
\hline Centro-oeste & 1.777 & 84,0 & 339 & 16,0 & 2.116 & 100,0 \\
\hline Total & 19.206 & 68,5 & 8.850 & 31,5 & 28.056 & 100,0 \\
\hline
\end{tabular}

Fonte: elaboração própria.

\section{Tabela 3}

Distribuição de equipes e níveis de assistência para saúde da mulher e da criança (Programa Nacional de Melhoria do Acesso e da Qualidade da Atenção Básica - PMAQ). Brasil, 2013.

\begin{tabular}{|c|c|c|c|c|c|c|}
\hline \multirow[t]{3}{*}{ Dimensão } & \multicolumn{6}{|c|}{ Nível da assistência } \\
\hline & \multicolumn{2}{|c|}{ Baixo } & \multicolumn{2}{|c|}{ Alto } & \multicolumn{2}{|c|}{ Total } \\
\hline & $\mathbf{n}$ & $\%$ & $\mathbf{n}$ & $\%$ & $\mathbf{n}$ & $\%$ \\
\hline Saúde da mulher & 19.777 & 70,5 & 8.279 & 29,5 & 28.056 & 100,0 \\
\hline Saúde da criança & 10.243 & 36,5 & 17.813 & 63,5 & 28.056 & 100,0 \\
\hline
\end{tabular}

Fonte: elaboração própria. 
Tabela 4

Regressão logística múltipla entre o nível de coordenação do cuidado das equipes de saúde da família e o nível de assistência para a saúde da mulher e da criança (Programa Nacional de Melhoria do Acesso e da Qualidade da Atenção Básica - PMAQ). Brasil, 2013.

\begin{tabular}{|c|c|c|c|c|}
\hline Dimensão/Nível de coordenação & $\beta$ & Valor de $\mathbf{p}$ & OR & IC95\% \\
\hline \multicolumn{5}{|l|}{ Criança } \\
\hline Baixo & - & - & - & - \\
\hline Alto & 2,17 & 0,001 & 8,79 & 8,$13 ; 9,517$ \\
\hline \multicolumn{5}{|l|}{ Mulher } \\
\hline Baixo & - & - & - & - \\
\hline Alto & 2,47 & 0,000 & 11,853 & 11,$132 ; 12,620$ \\
\hline
\end{tabular}

IC95\%: intervalo de 95\% de confiança; OR: odds ratio.

Fonte: elaboração própria.

Notas: Valor de Nagelkerke para saúde da mulher: 0,34; para saúde da criança: 0,23.

Os níveis de coordenação para a saúde da mulher e da criança foram ajustados por região geográfica e estrato.

\section{Discussão}

Os resultados encontrados neste estudo permitem uma visão panorâmica do perfil da coordenação do cuidado à saúde da mulher e da criança no Brasil. Um maior percentual de equipes se enquadrou em um nível baixo de coordenação no âmbito nacional, por estratos ou regiões, em que pesem algumas diferenças pontuais. Tais achados vão na direção de pesquisa desenvolvida por Souza et al. 19 que, a partir de dados do primeiro ciclo do PMAQ, identificaram uma maior frequência de equipes classificadas em níveis baixos de coordenação, com um maior nível relacionado a maior frequência de contato entre especialistas e profissionais da atenção básica, prontuário eletrônico integrado, uso e participação em telessaúde, apoio de especialistas da rede e fluxos institucionais de comunicação.

Os resultados encontrados neste trabalho corroboram a outros estudos de avaliação da APS que utilizaram o PCAtool como metodologia e apontam para limitações para a coordenação do cuidado. Carneiro et al. 21 encontraram fragilidades no processo de comunicação entre os profissionais e prestadores de serviços especializados. Turci et al. 22, ao avaliarem o desempenho da APS em Belo Horizonte, Minas Gerais, verificaram bons resultados para a coordenação do cuidado, contudo o atributo acesso, que está diretamente relacionado, apresentou baixa pontuação.

Nessa mesma perspectiva, em estudo desenvolvido em Londrina, Paraná, a coordenação do cuidado apresentou baixos escores no que se refere à transferência de informação entre o profissional de saúde e o usuário, no interesse do especialista no cuidado e no acesso do usuário ao prontuário, evidenciando fragilidade, sobretudo a prestação do cuidado integral 23. Em trabalho recente também realizado em Londrina, a coordenação do cuidado, quando avaliada isoladamente, apresentou altos escores, contudo foi identificada necessidade de melhora na qualidade das informações, na implantação de prontuários eletrônicos, na constituição das redes de atenção e na otimização de recursos da rede para assegurar os mecanismos de comunicação 24 .

Trabalhos nacionais que avaliaram a atenção à saúde na perspectiva dos usuários segundo os atributos da APS demonstraram resultados divergentes no Ceará 21 e no Espírito Santo 25. Em Minas Gerais 26, o atributo recebeu avaliação desfavorável e, em São Paulo, o atributo foi avaliado positivamente 27. Apesar do presente estudo não considerar a visão dos usuários sobre as ações de coordenação do cuidado, é importante considerar esse ponto de vista, uma vez que pode refletir a ruptura no processo de cuidado entre os pontos da rede 21.

$\mathrm{Na}$ análise dos estratos, houve predomínio do nível baixo de coordenação, e o comportamento de ambos os níveis foi diretamente proporcional à classificação do estrato, ou seja, o nível baixo aumentou nos estratos relativos a municípios de baixa densidade populacional, e os níveis mais altos foram 
superiores nos estratos correspondentes a municípios com alta densidade populacional. Os resultados deste estudo podem ser comparados aos encontrados no trabalho de Lima et al. ${ }^{20}$ que, ao analisar a efetivação dos atributos da APS nas regiões de saúde, segundo a tipologia desenvolvida pela pesquisa Regiões e Redes, verificou que os piores cenários para a coordenação do cuidado foram ligados à região 1, que corresponde aos municípios com baixa densidade socioeconômica e baixa oferta de serviços, e à região 2, que são aqueles municípios com média/alta densidade socioeconômica e baixa oferta de serviços.

Os achados deste estudo vão de encontro com os resultados obtidos por Fausto et al. 17, com dados do primeiro ciclo do PMAQ, ao verificar que, quanto maior o porte populacional das cidades, mais alto o percentual de respostas positivas para o uso de dispositivos facilitadores da coordenação assistencial, como prontuários eletrônicos, sistemas de agendamento de consultas e central de regulação. No entanto, o mesmo estudo verificou que, quanto menores as cidades, maiores as possibilidades de interação entre os profissionais da atenção primária e especializada, bem como a utilização de recursos como o telessaúde e o telemedicina, podendo esta ser uma explicação aos achados do presente trabalho.

A maior disponibilidade de serviços especializados nas regiões mais populosas, aliada a uma maior concentração de tecnologias de informação e serviços de média e alta complexidade, poderia facilitar a transmissão das informações e a transferência de cuidados entre os prestadores, favorecendo a coordenação assistencial. Estudo desenvolvido em área de vulnerabilidade social, onde se concentram municípios com baixa densidade populacional e baixo IDH (Índice de Desenvolvimento Humano), sinaliza para a necessidade de políticas que busquem promover uma maior articulação entre os serviços e que sejam capazes de trabalhar com as diversidades regionais fortemente encontradas no Brasil, contribuindo para reduzir a fragmentação na administração e no cuidado 28 .

Tais resultados também podem indicar a importância dos dispositivos de tecnologia de informação para as regiões menos favorecidas, na medida em que facilitam a comunicação entre os prestadores e as trocas de informação, contribuindo para uma melhor qualidade dos serviços, maior acesso e oportunidade aos usuários, reduzindo filas de espera para tratamentos especializados e promovendo maior agilidade nos diagnósticos 29 . Outro aspecto a ser considerado é que os municípios de baixa densidade populacional e baixa pontuação, em sua maioria, estão localizados nas regiões mais pobres do país, onde existe dificuldade de fixação de profissionais da saúde, de acesso a medicamentos e de serviços especializados. Tais fatores foram apontados por gestores, em estudo desenvolvido em três municípios nordestinos, como dificultadores do processo de cuidado, comprometendo a qualidade e a resolubilidade da atenção 30 .

Galvão et al. 28 debatem esse tema ao estudar a distribuição de serviços de saúde no Vale do Jequitinhonha, Minas Gerais, uma vez que verificaram a dificuldade dos municípios de pequeno porte em manter a oferta dos serviços, devido à escassez de recursos financeiros e administrativos, além da influência das diferenças socioeconômicas na articulação do sistema de saúde, constituindo barreiras para a coordenação assistencial.

Considerando o nível de coordenação nas regiões, identificou-se o predomínio de equipes com baixo nível de coordenação nas regiões Norte e Centro-oeste, e com alto nível no Sudeste e Nordeste, respectivamente. Tendo em vista que a existência de uma rede de atenção em saúde integrada refletese na qualidade da coordenação assistencial, por favorecer a continuidade da atenção entre profissionais e serviços 31 , os resultados do presente estudo chamam a atenção e corroboram os encontrados por Chaves et al. 32 que, ao analisarem a integração da rede assistencial, verificaram um maior percentual de equipes com baixo nível de desempenho na Região Norte e com alto nível na Região Sul.

Destaca-se que o predomínio do baixo nível de coordenação indica ainda uma incipiência desse atributo, provavelmente devido à dificuldade na transmissão de informações e no amadurecimento das redes de assistência nas regiões 33. A Região Norte, ao apresentar a maior proporção de equipes em baixo nível, assinala carência de iniciativas de coordenação nessa região, indicando a necessidade de estudos que compreendam quais fatores poderiam estar desencadeando esse comportamento, bem como quais os pontos frágeis da coordenação.

Com relação ao nível assistencial, para a saúde da mulher, prevaleceu o baixo nível, ao passo que, para a saúde da criança, a maior quantidade das equipes apresentou nível alto. Os achados para a qualidade da assistência à saúde da mulher são preocupantes e vão ao encontro dos encontrados por 
Tomasi et al. ${ }^{34}$, que registraram baixo percentual de mulheres com pré-natal adequado. Em publicação recente, a partir de dados do $2^{\circ}$ ciclo do PMAQ, a qualidade do cuidado pré-natal foi avaliada como nacionalmente inadequada, com pior cenário na região Norte, sobretudo devido à infraestrutura precária da rede, que compromete a qualidade do acesso e da assistência 33 . São lentos também os progressos na redução da mortalidade materna. Fatores como a ilegalidade do aborto, falhas na atenção ao parto, a peregrinação das gestantes pelos serviços e as altas taxas de cesariana ainda são reais no Brasil e contribuem para a manutenção das altas taxas de mortalidade materna 35 .

Já a evidência de maior quantidade de equipes com altos níveis de assistência à saúde da criança evidencia o resultado dos investimentos realizados na área, ao longo dos anos, no país. A criação de programas como o Programa Nacional de Imunização (PNI), o Programa de Atenção Integral à Saúde da Criança (PAISC) e posteriormente a implementação da Estratégia Saúde da Família (ESF), que ampliou a acesso aos serviços de APS, incrementando ações de prevenção e promoção, impactaram diretamente na redução da mortalidade infantil 35. Outras iniciativas como o Programa Nacional de Humanização do Parto e Nascimento e a Política Nacional de Atenção Humanizada ao Recém-nascido de Baixo Peso - Método Canguru, que buscaram melhorias na qualidade do parto e do nascimento, alcançaram êxito no aleitamento materno 36,37. Além desses, o desenvolvimento de programas intersetoriais, como o Bolsa Família, contribuiu para a redução da pobreza e, consequentemente, melhor qualidade de vida para as crianças 38 .

No que se refere à associação entre coordenação do cuidado e o resultado da qualidade da assistência à saúde da mulher e da criança no PMAQ, observou-se que existe uma associação positiva para ambos, demonstrando a importância da coordenação para a qualidade da assistência. Na visão de usuários, essa relação foi comprovada em estudo qualitativo no Sul do Brasil, onde a ausência desse atributo comprometeu a resolubilidade na atenção a crianças 39 . Ferreira e colaboradores também perceberam essa mesma influência positiva na atenção para crianças 40 .

A importância da continuidade assistencial para a saúde da mulher foi identificada como primordial no tratamento de câncer de colo uterino, e mecanismos como protocolos, apoio matricial, sistemas de referência e contrarreferência foram indicados para a garantia desse atributo 41 . Tais aspectos indicam que a coordenação do cuidado para a saúde da mulher e da criança é um ponto crucial para que ocorram a continuidade do cuidado e a resolubilidade da assistência. A utilização de mecanismos para comunicação efetiva entre profissionais e serviços, com a integração entre os pontos de atenção, garantindo uma eficiente transferência de informações, deve ser incentivada 3.

Uma das limitações deste estudo advém da amplitude do conceito de coordenação 13 e sua confluência com vários aspectos do processo de cuidado que, se misturando a outros atributos, pode conduzir a viés de informação. Além disso, é necessário pontuar que poderia ocorrer viés de seleção devido à adesão voluntária das equipes. A amostra, porém, inclui 90\% das equipes no país, reduzindo essa possibilidade.

\section{Conclusão}

O aprimoramento das atividades de coordenação do cuidado e a compreensão de como estão sendo realizadas é extremamente relevante. É um atributo que intrinsecamente interage com os demais, interfere na qualidade da assistência, podendo ser o diferencial para uma atenção ampla e eficaz.

Os resultados aqui apresentados permitem identificar que possuir um alto nível de coordenação do cuidado favorece um alto nível de qualidade da assistência à saúde da mulher e da criança. Considerando estratos e regiões, foi possível constatar um predomínio de baixos níveis de coordenação do cuidado nos serviços de APS, segundo dados do PMAQ, bem como de baixos níveis de qualidade da assistência à saúde da mulher, refletindo a necessidade de ações coordenadas nessa área. Na saúde da criança, obteve-se alto nível de qualidade da assistência, resultado dos investimentos, sobretudo para a redução da mortalidade infantil e garantia de crescimento e desenvolvimento saudáveis. Contribuíram significativamente para esse avanço a implementação de diversos programas que ampliaram o acesso aos serviços de APS, bem como o desenvolvimento de programas intersetoriais. Identificou-se também uma associação positiva entre equipes que apresentaram altos níveis de coordenação e altos níveis de qualidade da assistência, tanto para a saúde da mulher como da criança. 
Os resultados encontrados vão ao encontro de outros estudos realizados que verificaram incipiência do atributo, alertando para a necessidade de ações em âmbito nacional, estadual e municipal que favoreçam as atividades de coordenação do cuidado, além do desenvolvimento de pesquisas mais direcionadas ao entendimento dos elementos específicos desse atributo e dos nós críticos carentes de intervenção. No tocante à saúde da mulher, os achados representam a existência de pontos de fragilidade no sistema de saúde que favorecem a descontinuidade do cuidado e a consequente falha no cumprimento das metas nessa área, principalmente no que concerne à redução da mortalidade materna, ao controle do câncer e ao planejamento familiar.

A originalidade da abordagem de um tema complexo conjugado a um assunto de relevância no contexto sócio-político-cultural permitiu identificar lacunas e direcionar ao entendimento das limitações que comprometem o processo de cuidado.

\section{Colaboradores}

M. J. B. Cruz, A. F. Santos, L. H. L. Araújo e E. I. G. Andrade contribuíram na concepção e projeto; análise e interpretação dos dados; redação do artigo, revisão crítica relevante do conteúdo intelectual; aprovação final da versão a ser publicada; e na responsabilidade por todos os aspectos do trabalho, na garantia da exatidão e integridade de qualquer parte da obra.

\section{Informações adicionais}

ORCID: Maria Jesus Barreto Cruz (0000-00032735-3909); Alaneir de Fátima dos Santos (00000002-7674-0449); Lucas Henrique Lobato de Araújo (0000-0001-8528-3166); Eli Iola Gurgel Andrade (0000-0002-0206-2462).

\section{Agradecimentos}

O presente trabalho foi realizado com apoio da Coordenação de Aperfeiçoamento de Pessoal de Nível Superior - Brasil (Capes) - Código de Financiamento 001.

\section{Referências}

1. Bousquat A, Giovanella L, Campos EMS, Almeida PF, Martins CL, Mota PHS, et al. Atenção primária à saúde e coordenação do cuidado nas regiões de saúde: perspectiva de gestores e usuários. Ciênc Saúde Colet 2017; 22:1141-54.

2. Kringos DS, Boerma WGW, Hutchinson A, Saltman RB, editors. Building primary care in a changing Europe. Geneva: World Health Organization/European Observatory on Health Systems and Policies; 2015.

3. Santos NCCB, Vaz EMC, Nogueira JA, Toso BRGO, Collet N, Reichert APS. Presença e extensão dos atributos de atenção primária à saúde da criança em distintos modelos de cuidado. Cad Saúde Pública 2018; 34:e00014216.

4. Oliveira VBCA, Veríssimo MLOR. Children's health care assistance according to their families: a comparison between models of Primary Care. Rev Esc Enferm USP 2015; 49:30-6.

5. World Health Organization. Primary health care: now more than ever. Geneva: World Health Organization; 2008.

6. Terraza Núñez R, Vargas Lorenzo I, Vázquez Navarrete ML. La coordinación entre niveles asistenciales: una sistematización de sus instrumentos y medidas. Gac Sanit 2006; 20:48595.

7. Aleluia IRS, Medina MG, Almeida PF, Vilasbôas ALQ. Coordenação do cuidado na atenção primária à saúde: estudo avaliativo em município sede de macrorregião do nordeste brasileiro. Ciênc Saúde Colet 2017; 22:184556.

8. McDonald KM, Schultz E, Albin L, Pineda N, Lonhart J, Sundaram V, et al. Care coordination measures atlas; 2014. https://www. ahrq.gov/sites/default/files/publications/files/ ccm_atlas.pdf (acessado em 26/Jan/2018).

9. Almeida PF, Medina MG, Fausto MCR, Giovanella L, Bousquat A, Mendonça MHM. Coordenação do cuidado e Atenção Primária à Saúde no Sistema Único de Saúde. Saúde Debate 2018; 42:244-60. 
10. Almeida PF, Giovanella L, Nunan BA. Coordenação dos cuidados em saúde pela atenção primária à saúde e suas implicações para a satisfação dos usuários. Saúde Debate 2012; 36:375-91.

11. Silva RM, Andrade LOM. Coordenação dos cuidados em saúde no Brasil: o desafio federal de fortalecer a atenção primária à saúde. Physis (Rio J.) 2014; 24:1207-28.

12. Schang L, Waibel S, Thomson S. Measuring care coordination: health system and patient perspectives. London: LSE Health; 2013.

13. Bynum JPW, Ross JS. A measure of care coordination? J Gen Intern Med 2013; 28:336-8.

14. Giovanella L, Mendonça MHM, Fausto MCR, Almeida PF, Bousquat A, Lima JG, et al. A provisão emergencial de médicos pelo Programa Mais Médicos e a qualidade da estrutura das unidades básicas de saúde. Ciênc Saúde Colet 2016; 21:2697-708.

15. Uchôa SAC, Arcêncio RA, Fronteira I, Coêlho AA, Martiniano CS, Brandão ICA, et al. Potential access to primary health care: what does the National Program for Access and Quality Improvement data show? Rev Latinoam Enferm 2016; 24:e2672.

16. Departamento de Atenção Básica, Secretaria de Atenção a Saúde, Ministério da Saúde. Manual instrutivo: saúde mais perto de você - acesso e qualidade; 2013. http://189.28.128.100/dab/ docs/portaldab//publicacoes/manual_instru tivo_PMAQ_AB2013.pdf (acessado em 15/ $\mathrm{Jan} / 2018$ ).

17. Fausto MCR, Giovanella L, Mendonça MHM, Seidi H, Gagno J. A posição da Estratégia Saúde da Família na rede de atenção à saúde na perspectiva das equipes e usuários participantes do PMAQ-AB. Saúde Debate 2014; 38:1333.

18. Ibañez N, Rocha JSY, Castro PC, Ribeiro MCSA, Forster AC, Novaes MHD, et al. Avaliação do desempenho da atenção básica no Estado de São Paulo. Ciênc Saúde Colet 2006; 11:683703.

19. Souza MF, Santos AFD, Reis IA, Santos MADC, Jorge AO, Machado ATGM, et al. Care coordination in PMAQ-AB: an Item Response Theory-based analysis. Rev Saúde Pública 2017; 51:87.

20. Lima JG, Giovanella L, Fausto MCR, Bousquat A. Qualidade da atenção básica por tipos de regiões de saúde. Novos Caminhos 2016; (12). http://www.resbr.net.br/wp-content/ uploads/2016/07/NovosCaminho12.pdf.

21. Carneiro MSM, Melo DMS, Gomes JM, Pinto FJM, Silva MGC. Avaliação do atributo coordenação da Atenção Primária à Saúde: aplicação do PCATool a profissionais e usuários. Saúde Debate 2014; 38:279-95.

22. Turci MA, Lima-Costa MF, Macinko J. Influência de fatores estruturais e organizacionais no desempenho da atenção primária à saúde em Belo Horizonte, Minas Gerais, Brasil, na avaliação de gestores e enfermeiros. Cad Saúde Pública 2015; 31:1941-52.
23. Souza GT, Alves BA, Tacla MTGM, Collet N Toso BRGO. Avaliação do princípio da coordenação na atenção primária à saúde da criança em Londrina-PR. Semina Ciênc Biol Saúde 2015; 36:39-46.

24. Araujo JP, Viera CS, Oliveira BRG, Gaiva MA, Rodrigues RM. Assessment of the essential attributes of Primary Health Care for children. Rev Bras Enferm 2018; 71 Suppl 3:1366-72.

25. Lima EFA, Sousa AI, Primo CC, Leite FMC, Lima RCD, Maciel ELN. An assessment of primary care attributes from the perspective of female healthcare users. Rev Latinoam Enferm 2015; 23:553-9.

26. Paula FA, Silva CCR, Santos DF, Martins-Filho OA, Andrade RA. Avaliação da atenção à saúde do adulto em um município-polo do Vale do Jequitinhonha (MG). Saúde Debate 2015; 39:802-14

27. Furtado MCC, Braz JC, Pina JC, Mello DF, Lima RAG. Assessing the care of children under one year old in Primary Health Care. Rev Latinoam Enferm 2013; 21:554-61.

28. Galvão EL, Bodevan EC, Santos DF. Análise da distribuição geográfica dos serviços de saúde no Vale do Jequitinhonha, Minas Gerais. Hygeia 2015 ; 11:32-44

29. Santos AF, Fonseca Sobrinho D, Araujo LL, Procópio CSD, Lopes ÉAS, Lima AMLD, et al. Incorporação de Tecnologias de Informação e Comunicação e qualidade na atenção básica em saúde no Brasil. Cad Saúde Pública 2017; 33:e00172815.

30. Almeida PF, Santos AM. Primary Health Care: care coordinator in regionalized networks? Rev Saúde Pública 2016; 50:80.

31. Almeida PF, Giovanella L, Mendonça MHM, Escorel S. Desafios à coordenação dos cuidados em saúde: estratégias de integração entre níveis assistenciais em grandes centros urbanos. Cad Saúde Pública 2010; 26:286-98.

32. Chaves LA, Jorge AO, Cherchiglia ML, Reis IA, Santos MAC, Santos AF, et al. Integration of primary care in the healthcare network: analysis of the components in the external evaluation of the PMAQ-AB. Cad Saúde Pública 2018; 34:e0201515.

33. Guimarães WSG, Parente RCP, Guimarães TLF, Garnelo L. Acesso e qualidade da atenção pré-natal na Estratégia Saúde da Família: infraestrutura, cuidado e gestão. Cad Saúde Pública 2018; 34:e00110417.

34. Tomasi E, Fernandes PAA, Fischer T, Siqueira FCV, Silveira DS, Thumé E, et al. Qualidade da atenção pré-natal na rede básica de saúde do Brasil: indicadores e desigualdades sociais. Cad Saúde Pública 2017; 33:e00195815.

35. Leal MC, Szwarcwald CL, Almeida PVB, Aquino EML, Barreto ML, Barros F, et al. Saúde reprodutiva, materna, neonatal e infantil nos 30 anos do Sistema Único de Saúde (SUS). Ciênc Saúde Colet 2018; 23:1915-28. 
36. Venancio SI, Almeida H. Método mãe canguru: aplicação no Brasil, evidências científicas e impacto sobre o aleitamento materno. J Pediatr (Rio J.) 2004; 80 Suppl 1:S173-80.

37. Lamy Filho F, Silva AAM, Lamy ZC, Gomes MASM, Moreira MEL; Grupo de Avaliação do Método Canguru, et al. Evaluation of the neonatal outcomes of the kangaroo mother method in Brazil. J Pediatr (Rio J.) 2008; 84:428-35.

38. Rasella D, Aquino R, Santos CA, Paes-Sousa R, Barreto ML. Effect of a conditional cash transfer programme on childhood mortality: a nationwide analysis of Brazilian municipalities. Lancet 2013; 382:57-64.
39. Silva RMM, Silva Sobrinho RA, Neves ET, Toso BRGO, Viera CS. Challenges in the coordination of children's healthcare. Ciênc Saúde Colet 2015; 20:1217-24.

40. Ferreira TLS, Souza AMG, Medeiros JSS, Andrade FB. Avaliação do atributo coordenação do cuidado em serviços de puericultura na atenção primária à saúde. Rev Ciênc Plur 2017; 3:98-107.

41. Silva MRF, Braga JPR, Moura JFP, Lima JTO. Continuidade assistencial a mulheres com câncer de colo de útero em redes de atenção à saúde: estudo de caso, Pernambuco. Saúde Debate 2016; 40:107-19. 


\section{Abstract}

The study aimed to identify the association between coordination of care and quality of healthcare for women and children in primary healthcare in Brazil. A cross-sectional study was performed with data from 30,523 teams that participated in the Program for Improvement in Access and Quality of Basic Care (PMAQ) in 2013. Logistic regression was performed, in which the dependent variable was quality of healthcare for women and children and the independent variable was level of coordination of care. The multivariate analysis included variables that presented $p<0.05$. The model's fit was assessed with the Hosmer-Lemeshow test. The study assessed the results of 28,056 teams that conducted activities in coordination and healthcare for women and children simultaneously. In Brazil, the largest percentage of teams displayed low levels of coordination (68.5\%). The highest levels of coordination were found in stratum 6 (57.2\%) and the lowest in stratum 1(78.5\%). Among the major geographic regions, the North of Brazil showed the highest percentage of teams with low coordination (89.1\%), while the Southeast had the most teams with high coordination (37.6\%). More than two-thirds (70.5\%) of the teams showed low quality of care in women's health, while $63.5 \%$ showed high level of care in children's health. High level of coordination is associated with high quality of care both in women's health $(O R=11.85)$ and children's health $(O R=8.79)$. The predominance of low levels of coordination of care in Brazil and low quality of healthcare for women reflect the need for coordinated action in this area.

Primary Health Care; Child Health; Women's Health; Health Care Quality Assurance; Health Services Research

\section{Resumen}

El objetivo fue identificar la asociación del atributo coordinación del cuidado con la calidad de la asistencia a la salud de la mujer y del nino en la atención primaria a la salud en Brasil. Se realizó un estudio transversal, basado en datos de 30.523 equipos que participaron en el Programa de Mejora del Acceso y de la Calidad de la Atención Básica en 2013. Se realizó un análisis de regresión logística, la variable dependiente fue el nivel de calidad de la asistencia a la salud de la mujer y del niño, y la independiente el nivel de coordinación del cuidado. El análisis multivariado consideró variables que presentaron $p<0,05$. El ajuste del modelo se realizó por el test de HosmerLemeshow. Se evaluaron los resultados de 28.056 equipos que realizaron actividad de coordinación $y$ de asistencia a la salud de la mujer y del niño simultáneamente. En Brasil, el mayor porcentaje de equipos presentó un nivel bajo de coordinación (68,5\%). Los niveles más altos de coordinación se encontraron en el estrato $6(57,2 \%)$ y los más bajos en el estrato 1 (78,5\%). Entre las regiones el Norte presentó el mayor porcentaje de equipos con bajo nivel de coordinación $(89,1 \%)$ y el Sudeste el mayor con alto nivel (37,6\%). Para el nivel de calidad de la asistencia a la salud de la mujer un 70,5\% de los equipos tenía bajo nivel, y en la salud del niño un 63,5\% con alto nivel. Poseer un alto nivel de coordinación está asociado a tener un alto nivel de calidad de la asistencia, tanto en la salud de la mujer $(O R=11,85)$, como en la salud del niño $(\mathrm{OR}=8,79)$. Fue posible constatar un predominio de bajos niveles de coordinación del cuidado, en Brasil, así como bajos niveles de calidad de la asistencia a la salud de la mujer, reflejando la necesidad de acciones coordinadas en esta área.

Atención Primaria de Salud; Salud del Niño;

Salud de la Mujer; Garantía de Calidad

de Atención de Salud; Investigación

sobre Servicios de Salud
Recebido em 08/Jan/2019

Versão final reapresentada em 11/Abr/2019

Aprovado em 24/Mai/2019 